\title{
Maintenance treatment with the immunomodulator MGN1703, a Toll-like receptor 9 (TLR9) agonist, in patients with metastatic colorectal carcinoma and disease control after chemotherapy: a randomised, double-blind, placebo-controlled trial
}

\author{
Hans-Joachim Schmoll • Burghardt Wittig • Dirk Arnold • \\ Jorge Riera-Knorrenschild • Dieter Nitsche · Hendrik Kroening • \\ Frank Mayer · Johannes Andel · Reinhard Ziebermayr · Werner Scheithauer
}

Received: 24 February 2014 / Accepted: 28 March 2014 / Published online: 10 May 2014

(C) The Author(s) 2014. This article is published with open access at Springerlink.com

\begin{abstract}
Purpose This phase II study evaluated the synthetic DNA-based immunomodulator and Toll-like receptor 9 agonist MGN1703 as maintenance treatment in metastatic colorectal carcinoma (mCRC).

Methods Fifty-nine patients with mCRC and disease control after standard first-line chemotherapy were randomised to MGN1703 $60 \mathrm{mg}(N=43)$ or placebo $(N=16)$.

Results The hazard ratio (HR) for the primary endpoint [progression-free survival (PFS) from the start of maintenance] was 0.56 (95\% CI $0.29-1.08 ; P=0.07)$ and 0.55
\end{abstract}

Electronic supplementary material The online version of this article (doi:10.1007/s00432-014-1682-7) contains supplementary material, which is available to authorized users.

H.-J. Schmoll $(\bowtie)$

Department of Internal Medicine IV, Oncology/Hematology,

University Clinic Halle (Saale), Center for Cell and Gene

Therapy, Martin Luther University Halle-Wittenberg,

Ernst-Grube-Str. 40, 06120 Halle, Germany

e-mail: hans-joachim.schmoll@medizin.uni-halle.de

\section{B. Wittig}

Foundation Institute Molecular Biology and Bioinformatics,

Freie Universitaet Berlin, Arnimallee 22, 14195 Berlin, Germany

D. Arnold

Department of Medical Oncology and Hematology, Tumor Biology Center of the Albert-Ludwigs-University, Breisacher Str. 117, 79106 Freiburg, Germany

J. Riera-Knorrenschild

Hematology, Oncology and Immunology, University Hospital of Giessen and Marburg, Baldingerstraßse 1, 35043 Marburg, Germany

D. Nitsche

Hematology, Oncology, Gastroenterology, Barmherziger

Schwestern Linz, Seilerstätte 4, 4010 Linz, Austria
(95\% CI 0.3-1.0; $P=0.04$ ) by independent and investigator review, respectively. MGN1703 significantly improved PFS measured from the start of induction therapy versus placebo on independent (HR 0.49; $95 \%$ CI 0.26-0.94; $P=0.03$ ) and investigator review (HR 0.50; $95 \%$ CI 0.31$1.02 ; P=0.02)$. Overall survival (OS) data remain immature (HR $95 \%$; $95 \%$ CI $0.3-1.5 ; P=0.29$ ) with $28 / 43$ patients alive after a medium follow-up of $>17$ months. Retrospective subgroup analysis showed a significant effect of MGN1703 on PFS versus placebo in patients with greater than median tumour size reduction and normalised carcinoembryonic antigen concentrations following induction therapy, and in patients with elevated activated NKT cells $\geq 3.08 \%$. Adverse events were mild to moderate

\section{H. Kroening}

Hämatologie und Onkologie, Schwerpunktpraxis für Hämatologie und Onkologie, Hasselbachplatz 2, 39104 Magdeburg, Germany

\section{F. Mayer}

Department of Internal Medicine II, University of Tuebingen Medical Center, Ottfried-Mueller-Str. 10, 72076 Tuebingen, Germany

J. Andel

Internal Medicine II, Hospital of Steyr, Sierninger Str. 170, Steyr, Austria

R. Ziebermayr

Interne Hämatologie mit Stammzeltransplantation und medizinischer Onkologie, Elisabethinen Linz, Fadingerstraße 1, 4020 Linz, Austria

W. Scheithauer

Department of Internal Medicine I and Comprehensive Cancer Center, Medical University of Vienna, Waehringer Güertel 18-20, 1090 Vienna, Austria 


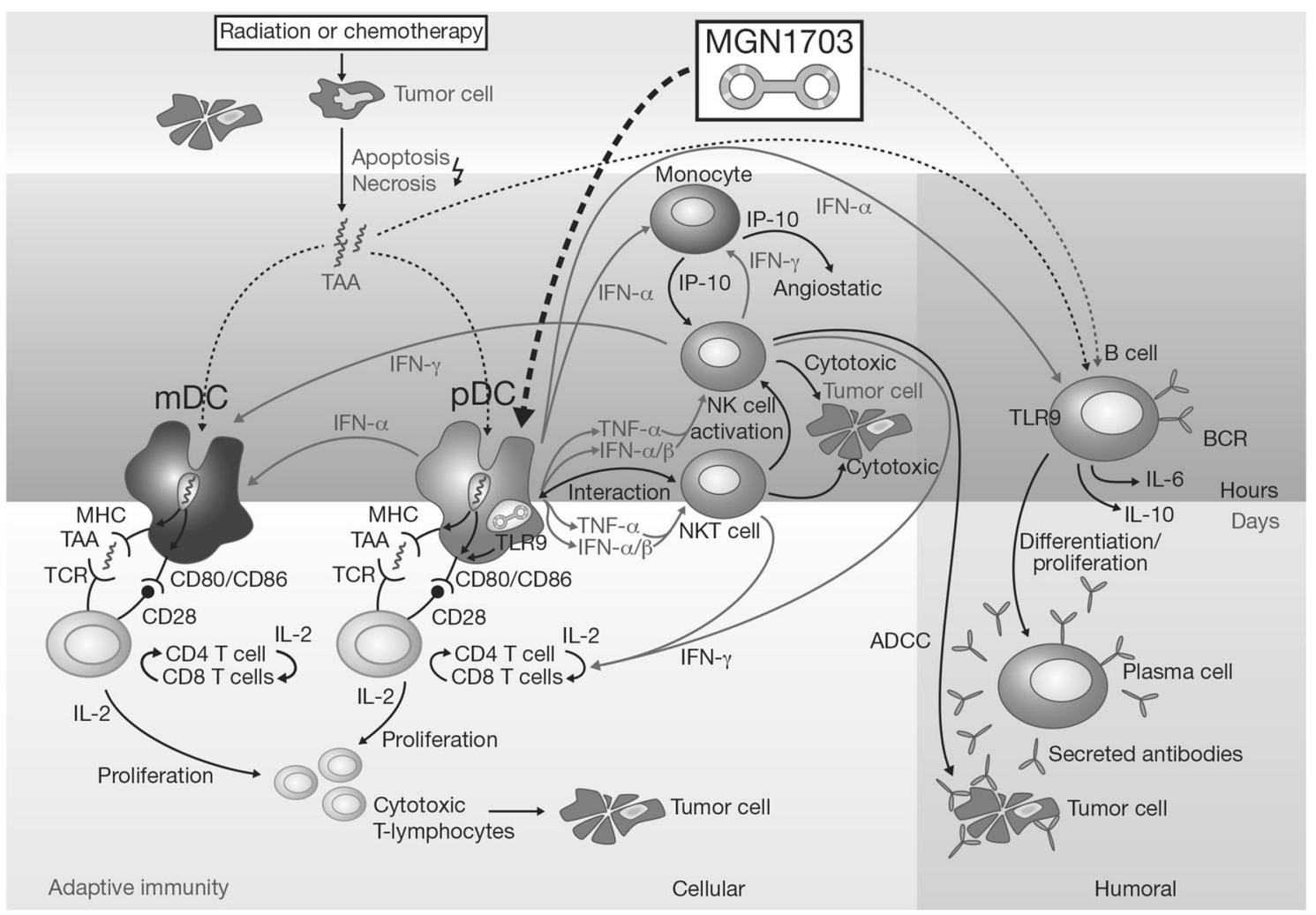

Fig. 1 Mechanism of action of MGN1703. ADCC antibody-dependent cell-mediated cytotoxicity, $B C R$ B-cell receptor, $I F N$ interferon, $I L$ interleukin, $m D C$ myeloid dendritic cells, $M H C$ major histocom- patibility complex, $N K$ cell natural killer cell, $N K T$ cell natural killer $\mathrm{T}$ cell, $p D C$ plasmacytoid dendritic cells, TAA tumour-associated antigens, TCR T-cell receptor, TLR9 Toll-like receptor 9 and limited to injection-site reactions or linked to general immune system activation.

Conclusions MGN1703 maintenance treatment was well tolerated and appears to induce durable and prolonged PFS and disease control in a subgroup of patients with mCRC following induction therapy. Activated NKT cells may be a predictive biomarker for selecting patients likely to benefit more from MGN1703.

Keywords MGN1703 - Immunomodulator · TLR9 agonist $\cdot$ Colorectal cancer $\cdot$ Maintenance

\section{Introduction}

In recent years, a number of agents have been developed with the aim of harnessing the inherent anti-cancer capabilities of the immune system. Most efforts have focused on the activation of adaptive immunity, as seen with ipilimumab-a cytotoxic $\mathrm{T}$ lymphocyte-associated antigen 4 inhibitor active in melanoma-and with other checkpoint inhibitors that target the programmed cell death 1 pathway (Hodi et al. 2010; Robert et al. 2011; Topalian et al. 2012; Tournigand et al. 2012; Brahmer et al. 2012). An additional immunomodulatory approach involves stimulation of innate immunity. MGN1703 is a novel, synthetic, dumbbell-shaped, covalently closed DNA molecule (Weihrauch et al., submitted), which activates the innate immune system via Toll-like receptor 9 (TLR9) (Fig. 1). The TLR9 receptor is expressed on plasmacytoid dendritic cells (pDC) and $\mathrm{B}$ cells and recognises non-methylated cytosine-guanine dinucleotide (CG) motifs in bacterial, viral and mitochondrial DNA (Schmidt et al. 2006a; Krieg 2002; Kanzler et al. 2007; Ahmad-Nejad et al. 2002). TLR agonists can stimulate innate antitumour mechanisms, including activation of natural killer T (NKT) cells, monocytes and macrophages, and induction of cytokines (Kanzler et al. 2007; Carpentier et al. 2006; Pashenkov et al. 2006; Friedberg et al. 2005). TLR agonists containing non-methylated CG motifs have exhibited limited, transient adverse effects and some efficacy in clinical trials in various tumour types (Kanzler et al. 2007; Carpentier et al. 2006; Pashenkov et al. 2006; Friedberg et al. 2005).

MGN1703 has been investigated as adjuvant therapy in two trials (Weihrauch et al. 2005; Wittig et al. 2001). In the first, MGN1703 was used in combination with chemoimmunotherapy in patients with metastatic colorectal carcinoma (CRC) (Weihrauch et al. 2005); in the second, 
patients with metastatic solid tumours of colon, renal cell, or melanoma origin received autologous gene-modified cells with adjuvant MGN1703 (Wittig et al. 2001). In both cases, regimens were well tolerated with mild, transient side effects, although MGN1703 doses were low (Weihrauch et al. 2005; Wittig et al. 2001). More recently, a phase I trial showed that twice-weekly subcutaneous applications of single-agent MGN1703 (up to $60 \mathrm{mg}$ ) were well tolerated in 28 patients with metastatic solid tumours (Weihrauch et al., submitted). Six patients had stable disease (prolonged in three cases), and one with refractory CRC had a partial response.

The current study evaluated MGN1703 as maintenance treatment in patients with metastatic CRC who had disease control after induction chemotherapy. Failure of a different TLR9 agonist to improve outcomes in advanced non-small cell lung cancer when added to chemotherapy suggests that the supportive immune response initiated by TLR9 agonists may firstly require release of tumour-associated antigens (Hirsh et al. 2011). A decreased tumour burden together with a chemotherapy-free interval allowing recovery of immune cells may also facilitate a more effective immune response (Hirsh et al. 2011). This type of immunotherapy may therefore prove successful when given sequentially after chemotherapy. Treatment for metastatic CRC has improved significantly over the last two decades (Chu 2012), and the potential value of maintenance therapy is being extensively investigated (Strickler and Hurwitz 2012; Díaz-Rubio et al. 2012; Tournigand et al. 2012; Koeberle et al. 2013; Koopman et al. 2014).

IMPACT, a randomised, placebo-controlled, doubleblind, phase II study, evaluated the efficacy and safety of subcutaneous MGN1703 (60 mg twice-weekly) as maintenance therapy following successful first-line induction therapy in metastatic CRC. This was the first placebocontrolled trial to prospectively investigate the impact of an immunomodulator as maintenance therapy in metastatic CRC and was based on the hypothesis that patients with disease control could benefit from immunotherapy.

\section{Methods}

\section{Study population}

The study recruited males or females aged over 18 years with histologically confirmed CRC that had been radiologically confirmed before starting first-line therapy as unresectable and advanced disease (American Joint Committee on Cancer stage IV). To be eligible, patients were also required to have previous first-line therapy with fluoropyrimidine plus irinotecan or oxaliplatin, with or without bevacizumab, for 4.5-6 months (treatment with oxaliplatin or irinotecan for at least 3 months); disease control after first-line therapy, defined as objective response or stable disease; at least one measurable lesion according to revised Response Evaluation Criteria in Solid Tumors (RECIST) (Eisenhauer et al. 2009); Eastern Cooperative Oncology Group (ECOG) performance status 0 or 1 ; and adequate bone marrow, liver and kidney function. Patients were excluded if they had tumour progression after first-line therapy; more than one previous line of systemic chemotherapy for metastatic CRC; known central nervous system metastases; history of autoimmune disease or immune deficiency; active or uncontrolled infections; transfusiondependent anaemia; concurrent chronic systemic immune therapy or immunosuppressant medication including steroid treatment; chemotherapy or immunotherapy within the 2 weeks before randomisation or radiotherapy 6 months before randomisation. All patients gave written, informed consent.

\section{Study design and treatments}

This randomised trial took place at 22 centres in Austria, France, Germany and Russia (ClinicalTrials.gov identifier: NCT01208194). The primary endpoint was progressionfree survival (PFS), measured from the date of randomisation to progression on maintenance therapy. Secondary endpoints included PFS measured from the start of induction therapy to progression on maintenance; overall survival (OS) from randomisation and start of induction therapy; objective response rate per RECIST during maintenance treatment; safety; and biomarkers for efficacy, including immunological response. Patients were randomised in a 2:1 ratio to MGN1703 $60 \mathrm{mg}$ or placebo, both given subcutaneously twice-weekly until disease progression, unacceptable toxicity, appearance of exclusion criteria, withdrawal of patient consent, or death. The study was approved by the relevant Investigational Review Boards or Ethics Committees and was run in accordance with the Declaration of Helsinki and Good Clinical Practice Guidelines.

\section{Randomisation and masking}

A randomisation list was generated using a standard computer programme and was designed in blocks of six patients. Treatment was allocated centrally; the sponsor provided sites with a patient number, which uniquely identified the treatment to be administered. The study was double-blind, with one copy of the complete randomisation code held by Mologen AG until study end and a second provided to a Data Safety Monitoring Board (DSMB). Investigators received a sealed random code envelope for each individual patient number, which was opened only in an emergency. 


\section{Assessments}

Tumour response was assessed by computed tomography scan using the revised RECIST (Eisenhauer et al. 2009) at baseline, at weeks 12, 18 and 24, and every 12 weeks thereafter. Assessment of scans by local investigators was confirmed by review by two independent radiologists. Regular safety assessments included physical examination, haematology and clinical chemistry tests, vital signs, electrocardiogram and ECOG performance status. Adverse events were monitored regularly and graded using the National Cancer Institute Common Toxicity Criteria for Adverse Events version 4.0.

Immunotherapy biomarker study

Whole blood samples were collected for the analysis of immunotherapy biomarkers at baseline and after 3, 6, 12 and 24 weeks, or at the end of study visit if after 24 weeks. Blood samples were shipped to the central laboratory (Labor Berlin $\mathrm{GmbH}$, Germany) at room temperature and were analysed by fluorescence activated cell sorting (FACS) within $24 \mathrm{~h}$. Frequency and activation status of the following immune cells were assessed by FACS: monocytes, B lymphocytes, T lymphocytes, NKT cells, NK cells, plasmacytoid dendritic cells and myeloid dendritic cells.

\section{Data analyses}

Sample size was estimated using Nquery version 7.0; the calculation assumed that PFS would increase from 3 months with placebo to 6 months with MGN1703, that at 6 months, the hazard ratio (HR) for progression would be 0.5 and an alpha level of 0.05 with $80 \%$ power. An estimated 129 patients were required (86 and 43 patients randomised to MGN1703 and placebo, respectively).

The primary analysis of efficacy endpoints was on an intent-to-treat basis. Efficacy was also analysed in a subgroup based on the per-protocol population but which excluded patients with abnormal prognostic laboratory parameters at baseline and/or the first visit on study. Safety analyses used the as-treated population (all patients who received at least one dose of study medication, with treatment assignments designated according to the actual study treatment received). The DSMB monitored safety data and advised on whether the trial could be continued. One interim analysis was planned after 60 patients had been treated for 4.5 months. PFS and OS were estimated using Kaplan-Meier methods and medians for each variable presented with $95 \%$ confidence intervals (CIs). PFS and OS were compared between treatment groups using the logrank test. The proportion of patients in each group who achieved an objective tumour response, defined as the sum of complete and partial responses, was calculated.
To identify patient subgroups most likely to obtain benefit from MGN1703 with respect to PFS, pre-planned analyses of immunotherapy biomarkers and patient characteristics at baseline were performed. Cox regression analysis and receiver operating characteristic analysis were used to identify biomarkers and optimal cut-off levels appropriate for the comparison of PFS. Methodology of the baseline characteristics analysis is described in the Appendix.

Role of the funding source

The study sponsor participated in study design and data collection and interpretation. This report was written by the corresponding author with contribution and review by all co-authors, including those employed by the sponsor. The corresponding author had full access to all study data and had final responsibility for the decision to submit the report for publication.

\section{Results}

Patients, demographics and baseline characteristics

Between June 2010 and May 2012, 59 patients were randomised to receive $\mathrm{MGN} 1703(N=43)$ or placebo $(N=16)$ (Fig. 2). Patient baseline demographics and characteristics were generally well balanced between treatment groups (Table 1).

Following a planned interim analysis, recruitment was halted in May 2012 before reaching the recruitment target of 129 patients. The primary reason for stopping the trial was the slow recruitment, which could not be accelerated, despite several attempts by the sponsor.

At the time of this analysis, median duration of followup for the MGN1703 group was 17.7 months (95\% CI

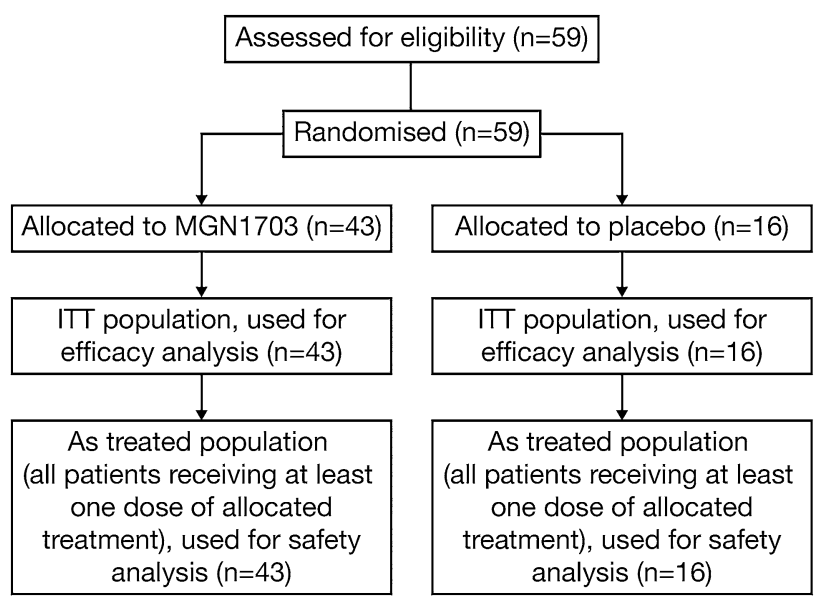

Fig. 2 Trial profile. ITT intent to treat 
Table 1 Patient demographic and disease characteristic at baseline (intent-to-treat cohort)

\begin{tabular}{lcc}
\hline Characteristic & $\begin{array}{c}\text { MGN1703 } \\
(N=43)\end{array}$ & $\begin{array}{c}\text { Placebo } \\
(N=16)\end{array}$ \\
\hline Age, median (range) & $65.0(39-80)$ & $67.5(44-80)$ \\
Gender: male/female, $n(\%)$ & $21 / 22(49 / 51)$ & $8 / 8(50 / 50)$ \\
ECOG performance status: 0/1, $n(\%)$ & $29 / 14(67 / 33)$ & $10 / 6(63 / 37)$ \\
Site of primary tumour, $n(\%)$ & & \\
Colon & $24(56)$ & $9(56)$ \\
Rectum & $13(30)$ & $6(17)$ \\
Both & $6(14)$ & $1(6)$ \\
Site of metastatic disease ${ }^{\mathrm{a}}, n(\%)$ & & \\
Liver only & $15(35)$ & $6(38)$ \\
Lung only & $4(9)$ & $1(6)$ \\
Other & $24(56)$ & $9(56)$ \\
Surgery: primary in situ & $12(28)$ & $5(31)$ \\
Median duration (range) of induction & $5.4(3-10)$ & $5.3(4-7)$ \\
therapy, months & & \\
Prior induction regimen, $n(\%)$ & & $7(44)$ \\
FOLFOX/XELOX + bevacizumab & $16(37)$ & $8(50)$ \\
FOLFIRI/XELIRI + bevacizumab & $21(49)$ & $1(6)$ \\
FOLFOX/XELOX alone & $6(14)$ & \\
Best response to induction therapy, $n(\%)$ & & $14(88)$ \\
CR/PR & $29(67)^{\mathrm{b}}$ & \\
SD & $13(30)$ & $2(12)$ \\
\hline
\end{tabular}

$C R$ complete response, ECOG Eastern Cooperative Oncology Group, $P R$ partial response, $S D$ stable disease

${ }^{a}$ Assessed before induction therapy

b Difference to $100 \%$ : missing values

13.8-19.6) and for placebo was 16.5 months (95\% CI 15.3-21.6). Median treatment duration was 3.2 months (range 1-26) and 2.9 months (range 1-12), respectively. All patients had discontinued; the most common reason for discontinuation was disease progression, reported in $31(72 \%)$ and $14(88 \%)$ patients in the MGN1703 and placebo groups, respectively. Eight patients discontinued study for clinical signs of relapse (such as fever, increasing biomarkers and local obstruction) that could not be confirmed by the independent review. Four patients continued to receive treatment with MGN1703 following the study.

\section{Efficacy and response}

Hazard ratio (HR) for investigator assessment of the primary endpoint was 0.55 (95\% CI $0.3-1.0 ; P=0.04)$, with MGN1703 maintenance treatment associated with durable PFS in some patients (Fig. 3a); median PFS was 2.8 months (95\% CI 2.8-4.1) with MGN1703 and 2.6 months (95\% CI 2.5-2.8) with placebo. HR for an independent review assessment of the primary endpoint was $0.56(95 \% \mathrm{CI}$ $0.29-1.08 ; P=0.07$ ) (see Fig. 3 footnote).
When measured from the start of induction therapy, PFS was significantly improved with MGN1703 versus placebo both with independent and investigator assessment (HR $0.49 ; 95 \%$ CI $0.26-0.94 ; P=0.03$ and HR $0.50 ; 95 \%$ CI $0.31-1.02 ; P=0.02$; Fig. $3 \mathrm{~b}$ and footnote). HR for PFS from randomisation in the protocol-defined subgroup population with 'good risk' characteristics was 0.52 (95\% CI $0.26-1.04 ; P=0.0526)$, corresponding to a median PFS of 3.0 months (95\% CI 2.8-6.6) with MGN1703 $(N=38)$ and 2.8 months (95\% CI 2.5-2.8) with placebo $(N=15)$.

Overall survival (OS) data were still immature after a median follow-up of over 17 months (Appendix Fig. S1a), with more than $50 \%$ of patients censored. Measured from randomisation, median OS was 22.6 months $(95 \% \mathrm{CI}$ 14.9-not reached [NR]) with MGN1703 and 15.1 months (95\% CI 10.6-NR) with placebo (HR 0.63; $95 \%$ CI 0.3$1.5 ; P=0.2886$; Appendix Fig. S1a). Median OS from the start of induction therapy was 26.3 months (95\% CI 21.0 NR) and 21.2 months (95\% CI 16.5-NR) for MGN1703 and placebo, respectively (HR $0.65 ; 95 \%$ CI $0.3-1.6$; $P=0.3339$; Appendix Fig. S1b).

Three patients in the MGN1703 group had a confirmed objective tumour response (objective response rate $7.0 \%$ ) during maintenance treatment, observed 3, 9 and 9 months after randomisation. At the time of this analysis, all three patients, plus a fourth patient in complete remission after induction chemotherapy, remain stable without relapse, having been on treatment for 16-30 months. One placebotreated patient had a response (objective response rate $6.3 \%$ ) after 3 months (response duration, 6 months).

Analyses of immunological biomarkers

Forty-six patients were included in a pre-planned analysis of potential immunological biomarkers ( 32 and 14 patients from the MGN1703 and placebo groups, respectively). In patients with high $(\geq 3.08 \%)$ activated NKT cell counts at baseline, MGN1703 was associated with significantly greater PFS than placebo (HR 0.27; $95 \%$ CI 0.14-0.82; $P=0.007$; Fig. 4a). This phenomenon was not observed in patients with activated NKT cell counts below this cut-off (HR 0.47; $95 \%$ CI 0.14-1.6; $P=0.16$; Fig. 4b).

Evidence for the activation of the immune system in patients treated with MGN1703 was provided by observed increases in the proportion of activated monocytes and pDC in these patients (Appendix).

Analyses of baseline patient characteristics predictive for progression-free survival

In the analyses of baseline patient characteristics, two of three factors included in the Cox proportional hazards model had statistically significant effects on PFS: reduction 

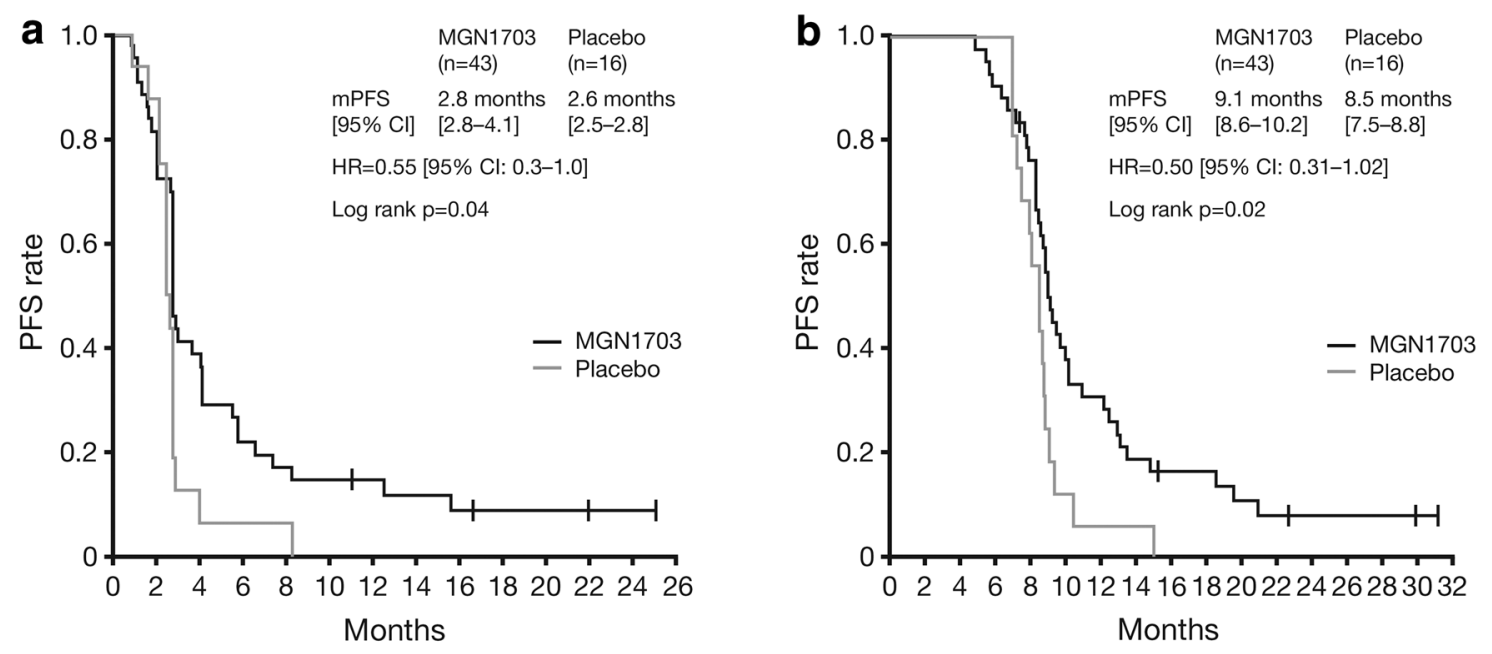

aSimilar results were obtained for the independent review assessment:

\begin{tabular}{|c|c|c|c|c|}
\hline & \multicolumn{2}{|c|}{$\begin{array}{c}\text { mPFS, } \\
\text { months }(95 \% \mathrm{Cl})\end{array}$} & \multirow{2}{*}{$\begin{array}{c}\mathrm{HR} \\
(95 \% \mathrm{Cl})\end{array}$} & \multirow[b]{2}{*}{$P$} \\
\hline & MGN1703 $(n=43)$ & Placebo $(n=16)$ & & \\
\hline $\begin{array}{l}\text { From } \\
\text { randomisation }\end{array}$ & $2.8(2.8-5.6)$ & $2.7(2.5-2.8)$ & $0.56(0.29-1.08)$ & 0.07 \\
\hline $\begin{array}{l}\text { From start of } \\
\text { induction therapy }\end{array}$ & $9.2(8.7-12.4)$ & $8.6(7.9-8.8)$ & $0.49(0.26-0.94)$ & 0.03 \\
\hline
\end{tabular}

Fig. 3 PFS by treatment group from a randomisation and $\mathbf{b}$ start of induction therapy (investigator assessment ${ }^{\mathrm{a}}$ ). $C I$ confidence interval, $H R$ hazard ratio, $m P F S$ median progression-free survival, $P F S$ progression-free survival
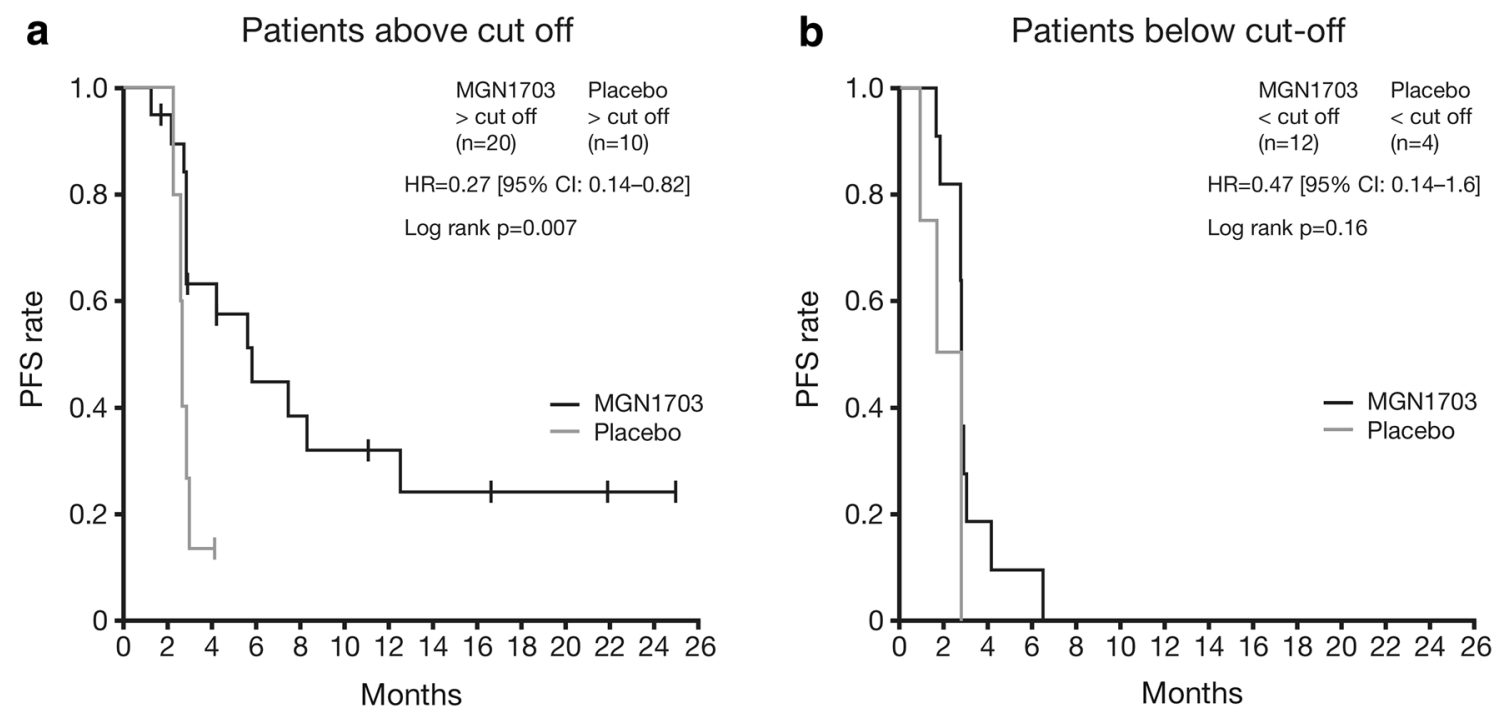

Fig. 4 PFS from randomisation by treatment group for patients with NKT cell counts $\geq 3.08 \%$ (a) or $<3.08 \%$ (b). CI confidence interval, $H R$ hazard ratio, $N K T$ natural killer T, $P F S$ progression-free survival

in tumour size at the end of induction therapy (>median versus $\leq$ median; HR 1.015 [95 \% CI 1.002-1.029; $P=0.0238])$ and carcinoembryonic antigen (CEA) concentration at the end of induction therapy (<upper limit of normal [ULN] versus $\geq$ ULN; HR 0.326 [95 \% CI 0.159$0.668 ; P=0.0022]$ ). Resection of primary tumour (yes versus no) was not significant (HR $0.851 ; 95 \%$ CI $0.385-$ 1.884; $P=0.6921)$. 
In patients with a reduction in tumour size following induction therapy greater than the median reduction, PFS was significantly longer with MGN1703 than placebo but this effect of MGN1703 was not observed in patients with a reduction in tumour size less than median (Appendix Fig. S2a). Similarly, in patients with CEA concentrations within the normal range, PFS was significantly longer with MGN1703 than placebo but no such effect was observed in patients with CEA above the normal range (Appendix Fig. S2b). PFS was also significantly extended with MGN1703 in patients with response after induction but not in patients with stable disease (Appendix Fig. S2c). These data should be treated with some caution, however, since patient numbers are small, particularly in the placebo group.

\section{Safety and tolerability}

In the MGN1703 and placebo groups, respectively, 79 and $38 \%$ of patients experienced any treatment-emergent adverse event; overall incidence of grade $3 / 4$ adverse events was 26 and $13 \%$, respectively. Treatment-related adverse events were reported in 33 and $12.5 \%$ of MGN1703- and placebo-treated patients, respectively, while grade $3 / 4$ adverse events regarded as treatment-related were rare (2 and $8 \%$, respectively; $n=1$ in each group). One patient discontinued MGN1703 because of an adverse event (sensory neuropathy; no discontinuations in the placebo group).

Grade 3/4 treatment-emergent adverse events observed in the MGN1703 group included ileus $(n=4)$, hypertension $(n=2)$, worsening of hypertension $(n=2)$, neutropenia, aspartate aminotransferase increase, sensory polyneuropathy and sepsis (each $n=1$ ). The case of polyneuropathy was considered possibly related to MGN1703, but was more likely to have been associated with previous oxaliplatin chemotherapy. Among five patients with serious adverse events, two had ileus, a third had ileus with sepsis and another had ileus with alimentary toxicosis; none of these events were considered related to treatment. The fifth patient was admitted to hospital with atypical pneumonia (grade 2), which was considered possibly related to MGN1703 treatment. In the placebo group, grade 3 adverse events comprised papular exanthema, food poisoning and coxarthrosis (each $n=1$ ); there were no grade 4 adverse events and none were considered serious.

Treatment-related adverse events of interest because of the mode of action of MGN1703 are summarised in Table 2 . These were typically mild to moderate (grade 1 or 2) and in the MGN1703 group included most commonly flu-like symptoms and injection-site reactions. Mild local reactions (pain, redness, itching or induration) occurred in 77 and $6 \%$ of patients in the MGN1703 and placebo groups, respectively. These symptoms were limited at the site of injection and did not result in treatment interruption.
Table 2 Summary of treatment-emergent adverse events of particular interest due to MGN1703 mechanism of action

\begin{tabular}{|c|c|c|c|c|}
\hline \multirow{2}{*}{$\begin{array}{l}\text { AE, number of } \\
\text { patients }(\%)\end{array}$} & \multicolumn{2}{|c|}{$\operatorname{MGN} 1703(N=43)$} & \multicolumn{2}{|c|}{ Placebo $(N=13)$} \\
\hline & Grade $1 / 2$ & Grade $3 / 4$ & Grade $1 / 2$ & Grade 3/4 \\
\hline Flu-like symptoms & $6(13.9)$ & - & $1(7.7)$ & - \\
\hline General pain & $4(9.3)$ & - & $1(7.7)$ & - \\
\hline $\begin{array}{l}\text { General rash, itching, } \\
\text { paraesthesia }\end{array}$ & $4(9.3)$ & - & - & $1(7.7)$ \\
\hline Injection-site reaction & $2(4.6)$ & - & $1(7.7)$ & - \\
\hline Atypical pneumonia & $2(4.6)$ & - & - & - \\
\hline Polyneuropathy & $1(2.3)$ & $1(2.3)$ & - & - \\
\hline ANA increased & $1(2.3)$ & - & $1(7.7)$ & - \\
\hline Fatigue & $1(2.3)$ & - & - & - \\
\hline Hypertension & $1(2.3)$ & - & - & - \\
\hline
\end{tabular}

$A E$ adverse event, $A N A$ antinuclear antibody

\section{Discussion}

The IMPACT trial showed that MGN1703 maintenance treatment was well tolerated and appears to induce durable PFS and disease control in selected patients with advanced CRC following induction therapy.

Findings from the study reflect the complications and challenges faced in assessing clinical response to immunotherapies. In the primary endpoint analysis, Kaplan-Meier curves separated after the median point; similar findings have been reported in other recent studies in metastatic $\mathrm{CRC}$, for example, in populations with mixed KRAS mutation status treated with epidermal growth factor receptor inhibitors (Saif and Shah 2009; Ramos et al. 2008). Therefore, the activity of MGN1703 may be limited to a specific subpopulation of patients who achieve a prolonged response to treatment. Indeed, results from the secondary analyses indicate that three possible subgroups existed. Firstly, the greatest benefit with MGN1703 was observed in patients who achieved shrinkage of their tumour after induction chemotherapy. This is consistent with the theory that immunomodulatory treatment is more likely to be successful in patients with relatively low tumour burden. Similarly, normal CEA values after induction therapy also had predictive value. Finally, results from the immunological biomarker analysis reported a significant effect of MGN1703 on PFS relative to placebo in patients with levels of activated NKT cells $\geq 3.08 \%$ but not in those with levels below this cut-off. Activated NKT cells may be a potential biomarker for selecting patients more likely to benefit from MGN1703 treatment.

Two assessments of response were used in the trial (investigator and independent review). Primary endpoint results with the two assessment methods were similar, although $P$-values decreased with the investigator assessment 
relative to independent review (0.04 versus 0.07 ), reflecting a decrease in statistical uncertainty caused by a reduction in the number of censored patients. In the independent review, 13 patients were censored (eight local relapses as assessed by investigators could not be confirmed on scans by the independent review). The investigator assessment included these eight patients so that only five patients were censored. Such inaccurate identification of relapse is indicative of the challenges involved in measuring responses to immunotherapy. Immunomodulators are associated with unique patterns of response versus conventional therapies. In studies of ipilimumab, for example, disease regression was sometimes not seen for several months and, in other cases, lesion growth was observed due to immune-related inflammation not disease progression (Weber et al. 2012).

Immune-related response criteria have now been defined (Wolchok et al. 2009), although traditional RECIST criteria, which may not detect all responses to immunotherapy (Hales et al. 2010), were used in the current trial. Therefore, in subsequent studies on MGN1703, immune-related response criteria may be more appropriate.

Data from several randomised trials investigating maintenance treatment for metastatic CRC with agents such as bevacizumab have recently been reported (Díaz-Rubio et al. 2012; Tournigand et al. 2012; Koeberle et al. 2013; Koopman et al. 2014). Interpretation of these data is complex, as all the trials differ in design and in how the maintenance or de-escalation phase is managed. Therefore, many questions remain regarding whether, and in what form, maintenance treatment should be used in this setting. Comparison of PFS data from the current trial and other trials of maintenance therapy is made difficult by the different assessment schedules employed. In the study reported here, the first assessment was performed at month 3 to allow patients to receive adequate immunomodulatory therapy; this is reflected in the steep decay of the upper portions of the Kaplan-Meier curves. With regard to other immunomodulators, the current data appear to be consistent with observations from some trials showing no effect in terms of median PFS but lasting benefit in some patients (Chung et al. 2010; Kirkwood et al. 2010). It may be of interest to observe, as with other immunomodulators, whether mature OS results for MGN1703 point to a broader activity than seen on the shorter-term PFS endpoint.

Structural and preclinical studies have shown that MGN1703 is a potent TLR9 agonist with limited capacity for interactions with molecules outside its target structure (Schmidt et al. 2006b, 2008; Kapp et al., submitted). In addition to this specificity, the relevant cellular activation profile of MGN1703 is distinct to that of other TLR9 agonists, such as PF-3512676, which failed to show benefit compared with chemotherapy alone in two phase III trials in NSCLC (Hirsh et al. 2011; Manegold et al. 2012). Additional immunotherapy approaches have been investigated in patients with tumour types other than CRC. These include agents that target specific immune regulatory checkpoints and increase the endogenous antitumour immune response. For example, ipilimumab which binds to CTLA-4, has been shown to result in 2-year survival rates of $23.5 \%$ in patients with metastatic melanoma (Hodi et al. 2010). The majority of adverse events with ipilimumab are consistent with the proposed mechanism of action and can be severe, and long-lasting (Hodi et al. 2010). Other immune checkpoint inhibitors have been developed to target the programmed cell death 1 receptor and prevent T-cell inactivation, with studies investigating their potential in melanoma and NSCLC (Pardoll and Drake 2012). The combination of MGN1703 with a checkpoint inhibitor could offer a potentially synergistic immunotherapeutic approach in this setting.

Safety data for MGN1703 were in accordance with those from the phase I trial (Weihrauch et al., submitted). MGN1703 was generally well tolerated, with most drugrelated adverse events being mild or moderate. Common adverse events included injection-site reactions, fever and fatigue. Good tolerance of MGN1703 was confirmed by the lack of treatment interruption despite therapy for up to 30 months in patients experiencing prolonged response. In general, the tolerability profile of MGN1703 appears similar to that of therapeutic vaccines such as sipuleucel-T, rather than the checkpoint inhibitors (Frohlich 2012).

Overall, the results from this trial are encouraging and suggest a potential role for TLR9-targeted immunoactivation with MGN1703 in maintenance therapy of metastatic $\mathrm{CRC}$ after an effective first-line regimen inducing a tumour response. It is important, however, that the current results are not over-interpreted because of the very limited sample size due to the early study termination. A randomised, phase III trial of MGN1703 in the first-line/maintenance setting in patients with metastatic CRC is planned.

Acknowledgments We thank Seren Communications for writing assistance with this manuscript, the funding for which was provided by Mologen AG.

Conflict of interest BW is listed as co-inventor on $\mathrm{dSLIM}^{\circledR}$ patents, has share ownership in Mologen AG and has received research funding from Mologen AG. JR-K received a travel grant from Mologen AG to present the IMPACT trial data at ASCO 2013. H-JS, DA, DN, HK, FM, JA, RZ, WS have no disclosures to declare.

Open Access This article is distributed under the terms of the Creative Commons Attribution License which permits any use, distribution, and reproduction in any medium, provided the original author(s) and the source are credited.

\section{References}

Ahmad-Nejad P, Häcker H, Rutz M, Bauer S, Vabulas RM, Wagner H (2002) Bacterial CpG-DNA and lipopolysaccharides activate Toll-like receptors at distinct cellular compartments. Eur J Immunol 32:1958-1968 
Brahmer JR, Tykodi SS, Chow LQ, Hwu WJ, Topalian SL, Hwu P, Drake CG, Camacho LH, Kauh J, Odunsi K, Pitot HC, Hamid O, Bhatia S, Martins R, Eaton K, Chen S, Salay TM, Alaparthy S, Grosso JF, Korman AJ, Parker SM, Agrawal S, Goldberg SM, Pardoll DM, Gupta A, Wigginton JM (2012) Safety and activity of anti-PD-L1 antibody in patients with advanced cancer. N Engl J Med 366:2455-2465

Carpentier A, Laigle-Donadey F, Zohar S, Capelle L, Behin A, Tibi A, Martin-Duverneuil N, Sanson M, Lacomblez L, Taillibert S, Puybasset L, Van Effenterre R, Delattre JY, Carpentier AF (2006) Phase 1 trial of a $\mathrm{CpG}$ oligodeoxynucleotide for patients with recurrent glioblastoma. Neuro Oncol 8:60-66

Chu E (2012) An update on the current and emerging targeted agents in metastatic colorectal cancer. Clin Colorectal Cancer 11:1-13

Chung KY, Gore I, Fong L, Venook A, Beck SB, Dorazio P, Criscitiello PJ, Healey DI, Huang B, Gomez-Navarro J, Saltz LB (2010) Phase II study of the anti-cytotoxic T-lymphocyteassociated antigen 4 monoclonal antibody, tremelimumab, in patients with refractory metastatic colorectal cancer. J Clin Oncol 28:3485-3490

Díaz-Rubio E, Gómez-España A, Massutí B, Sastre J, Abad A, Valladares M, Rivera F, Safont MJ, Martínez de Prado P, Gallén M, González E, Marcuello E, Benavides M, Fernández-Martos C, Losa F, Escudero P, Arrivi A, Cervantes A, Dueñas R, LópezLadrón A, Lacasta A, Llanos M, Tabernero JM, Antón A, Aranda E; Spanish Cooperative Group for the Treatment of Digestive Tumors (2012) First-line XELOX plus bevacizumab followed by XELOX plus bevacizumab or single-agent bevacizumab as maintenance therapy in patients with metastatic colorectal cancer: the phase III MACRO TTD study. Oncologist 17:15-25

Eisenhauer EA, Therasse P, Bogaerts J, Schwartz LH, Sargent D, Ford R, Dancey J, Arbuck S, Gwyther S, Mooney M, Rubinstein L, Shankar L, Dodd L, Kaplan R, Lacombe D, Verweij J (2009) New response evaluation criteria in solid tumours: revised RECIST guideline (version 1.1). Eur J Cancer 45:228-247

Friedberg JW, Kim H, McCauley M, Hessel EM, Sims P, Fisher DC, Nadler LM, Coffman RL, Freedman AS (2005) Combination immunotherapy with a CpG oligonucleotide (1018 ISS) and rituximab in patients with non-Hodgkin lymphoma: increased interferon-alpha/beta-inducible gene expression, without significant toxicity. Blood 105:489-495

Frohlich MW (2012) Sipuleucel-T for the treatment of advanced prostate cancer. Semin Oncol 39:245-252

Hales RK, Banchereau J, Ribas A, Tarhini AA, Weber JS, Fox BA, Drake CG (2010) Assessing oncologic benefit in clinical trials of immunotherapy agents. Ann Oncol 21:1944-1951

Hirsh V, Paz-Ares L, Boyer M, Rosell R, Middleton G, Eberhardt WE, Szczesna A, Reiterer P, Saleh M, Arrieta O, Bajetta E, Webb RT, Raats J, Benner RJ, Fowst C, Meech SJ, Readett D, Schiller JH (2011) Randomized phase III trial of paclitaxel/carboplatin with or without PF-3512676 (Toll-like receptor 9 agonist) as first-line treatment for advanced non-small-cell lung cancer. J Clin Oncol 29:2667-2674

Hodi FS, O'Day SJ, McDermott DF, Weber RW, Sosman JA, Haanen JB, Gonzalez R, Robert C, Schadendorf D, Hassel JC, Akerley W, van den Eertwegh AJ, Lutzky J, Lorigan P, Vaubel JM, Linette GP, Hogg D, Ottensmeier CH, Lebbé C, Peschel C, Quirt I, Clark JI, Wolchok JD, Weber JS, Tian J, Yellin MJ, Nichol GM, Hoos A, Urba WJ (2010) Improved survival with ipilimumab in patients with metastatic melanoma. N Engl J Med 363:711-723

Kanzler H, Barrat FJ, Hessel EM, Coffman RL (2007) Therapeutic targeting of innate immunity with Toll-like receptor agonists and antagonists. Nat Med 13:552-559

Kirkwood JM, Lorigan P, Hersey P, Hauschild A, Robert C, McDermott D, Marshall MA, Gomez-Navarro J, Liang JQ, Bulanhagui CA (2010) Phase II trial of tremelimumab (CP-675,206) in patients with advanced refractory or relapsed melanoma. Clin Cancer Res 39:245-252

Koeberle D, Betticher DC, Von Moos R, Dietrich D, Brauchli P, Baertschi D, Matter-Walstra K, Winterhalder RC, Borner MM, Anchisi S, Moosmann P, Kollar A, Saletti P, Roth A, Frueh M, Kueng M, Popescu RA, Schacher S, Hess V, Herrmann R (2013) Bevacizumab continuation versus no continuation after first-line chemo-bevacizumab therapy in patients with metastatic colorectal cancer: A randomized phase III noninferiority trial (SAKK 41/06). J Clin Oncol 31(suppl; abstr 3503)

Koopman M, Simkens L, May A, Mol L, van Tinteren H, Punt CJA, on behalf of the CAIRO3 Study Group (2014) Final results and subgroup analyses of the phase 3 CAIRO3 study: Maintenance treatment with capecitabine and bevacizumab versus observation after induction treatment with chemotherapy and bevacizumab in metastatic colorectal cancer (mCRC). J Clin Oncol 32(suppl 3; abstr LBA388)

Krieg AM (2002) CpG motifs in bacterial DNA and their immune effects. Annu Rev Immunol 20:709-760

Manegold C, van Zandwijk N, Szczesna A, Zatloukal P, Au JS, Blasinska-Morawiec M, Serwatowski P, Krzakowski M, Jassem J, Tan EH, Benner RJ, Ingrosso A, Meech SJ, Readett D, Thatcher N (2012) A phase III randomized study of gemcitabine and cisplatin with or without PF-3512676 (TLR9 agonist) as first-line treatment of advanced non-small-cell lung cancer. Ann Oncol 23:72-77

Pardoll D, Drake C (2012) Immunotherapy earns its spot in the ranks of cancer therapy. J Exp Med 209:201-209

Pashenkov M, Goëss G, Wagner C, Hörmann M, Jandl T, Moser A, Britten CM, Smolle J, Koller S, Mauch C, Tantcheva-Poor I, Grabbe S, Loquai C, Esser S, Franckson T, Schneeberger A, Haarmann C, Krieg AM, Stingl G, Wagner SN (2006) Phase II trial of a toll-like receptor 9-activating oligonucleotide in patients with metastatic melanoma. J Clin Oncol 24:5716-5724

Ramos FJ, Macarulla T, Capdevila J, Elez E, Tabernero J (2008) Understanding the predictive role of $\mathrm{K}$-ras for epidermal growth factor receptor-targeted therapies in colorectal cancer. Clin Colorectal Cancer 7(Suppl 2):S52-S57

Robert C, Thomas L, Bondarenko I, O’Day S, Weber J, Garbe C, Lebbe C, Baurain JF, Testori A, Grob JJ, Davidson N, Richards J, Maio M, Hauschild A, Miller WH Jr, Gascon P, Lotem M, Harmankaya K, Ibrahim R, Francis S, Chen TT, Humphrey R, Hoos A, Wolchok JD (2011) Ipilimumab plus dacarbazine for previously untreated metastatic melanoma. N Engl J Med $364: 2517-2526$

Saif MW, Shah M (2009) K-ras mutations in colorectal cancer: a practice changing discovery. Clin Adv Hematol Oncol 7:45-53, 64

Schmidt M, Anton K, Nordhaus C, Junghans C, Wittig B, Worm M (2006a) Cytokine and Ig-production by CG-containing sequences with phosphorodiester backbone and dumbbell-shape. Allergy 61:56-63

Schmidt M, Cristobal J, Sander A, Brzezicha B, König-Merediz SA, Wittig B (2006b) dSLIM immunomodulators induce anti-tumor responses both in vitro and in vivo. Mol Ther 13(suppl 1; abstr 426):S164

Schmidt M, Brzezicha B, Chen Y, König-Merediz SA, Wittig B (2008) dSLIM immunomodulators reduce tumor growth in various animal tumor models. Mol Ther 16(suppl 1; abstr 935):S1-S389

Strickler JH, Hurwitz HI (2012) Maintenance therapy for first-line metastatic colorectal cancer: activity and sustainability. Oncologist 17:9-10

Topalian SL, Hodi FS, Brahmer JR et al (2012) Safety, activity, and immune correlates of anti-PD-1 antibody in cancer. N Engl J Med 366:2443-2454

Tournigand C, Samson B, Scheithauer W, Lledo G, Viret F, Andre T, Ramée JF, Tubiana-Mathieu N, Dauba J, Dupuis O, Rinaldi Y, 
Mabro M, Aucoin N, Khalil A, Latreille J, Louvet C, Brusquant D, Bonnetain F, Chibaudel B, De Gramont A (2012) Bevacizumab (Bev) with or without erlotinib as maintenance therapy, following induction first-line chemotherapy plus Bev, in patients (pts) with metastatic colorectal cancer (mCRC): Efficacy and safety results of the International GERCOR DREAM phase III trial. J Clin Oncol 30(suppl; abstr LBA3500)

Weber JS, Kahler KC, Hauschild A (2012) Management of immunerelated adverse events and kinetics of response with ipilimumab. J Clin Oncol 30:2691-2697

Weihrauch MR, Ansén S, Jurkiewicz E, Geisen C, Xia Z, Anderson KS, Gracien E, Schmidt M, Wittig B, Diehl V, Wolf J, Bohlen H, Nadler LM (2005) Phase I/II combined chemoimmunotherapy with carcinoembryonic antigen-derived HLA-A2-restricted CAP-1 peptide and irinotecan, 5-fluorouracil, and leucovorin in patients with primary metastatic colorectal cancer. Clin Cancer Res 11:5993-6001

Wittig B, Märten A, Dorbic T, Weineck S, Min H, Niemitz S, Trojaneck B, Flieger D, Kruopis S, Albers A, Löffel J, Neubauer A, Albers P, Müller S, Sauerbruch T, Bieber T, Huhn D, SchmidtWolf IG (2001) Therapeutic vaccination against metastatic carcinoma by expression-modulated and immunomodified autologous tumor cells: a first clinical phase I/II trial. Hum Gene Ther 12:267-278

Wolchok JD, Hoos A, O’Day S, Weber JS, Hamid O, Lebbé C, Maio M, Binder M, Bohnsack O, Nichol G, Humphrey R, Hodi FS (2009) Guidelines for the evaluation of immune therapy activity in solid tumors: immune-related response criteria. Clin Cancer Res 15:7412-7420 